\title{
Erfolge nur mit den Patienten
}

\author{
Hansgeorg Ließem
}

Projekte der Integrierten Versorgung gemäß $§ 140$ a-d SGB $V$ gewinnen zunehmend auch für soziale Leistungserbringer an Bedeutung. In Niedersachsen beispielsweise sind inzwischen mehr als 50 ambulant ausgerichtete Psychiatrieprojekte mit den Krankenkassen vereinbart worden. Hierbei wurden Träger der Sozialpsychiatrie insbesondere über die Funktionen "Ambulante Psychiatrische Pflege " und »Soziotherapie « einbezogen. Die fachliche Diskussion rückt dabei verständlicherweise zunächst einmal die verschiedenen organisatorischen und wirtschaftlichen Ansatzmöglichkeiten für Integrierte Versorgung in den Mittelpunkt (1). Im Sinne der Kundenorientierung sollten jedoch die Interessen der Patienten zum Maßstab der Betrachtung gemacht werden.

Die Patienten in Deutschland erkennen immer stärker, dass sie nicht die Objekte des Gesundheitswesens sind, sondern dessen Subjekte. Ihre Behandlung finanzieren letztendlich nicht die Krankenkassen, sondern sie selbst. (2) Die Krankenkassen verwalten lediglich die Interessen ihrer Mitglieder als Ausdrucksform der Selbstverwaltung.

Aber auch die Krankheit selbst ist in jeder Hinsicht eine subjektive Erscheinung. Sie entwickelt sich in einer weitgehend individuell geprägten Lebenssituation. Sie kann dazu zwingen, den alltäglichen Lebensablauf zu ändern, was nicht immer zwangsläufig der Lebensqualität schadet. Krankheit kann, als Alarm- und Notsignal gedeutet, heilsam sein und notwendige Lebensänderungen ermöglichen. Die Überwindung der Krankheit ist immer ein Prozess, an dem der Erkrankte selbst regen Anteil nimmt.

In diesen Erkrankungs- und Heilungsprozess sind nicht nur der Patient selbst, sondern alle Menschen einbezogen, mit denen er in Kontakt steht. Einige fühlen mit ihm, unterstützen seine Selbstheilungskräfte. Andere suchen ihren Vorteil daraus zu ziehen (manchmal auch Professionelle des Gesundheitswesens oder Angehörige) oder lasten ihm seinen Krankheitszustand persönlich an. Diese Ablehnung kann bis zur Stigmatisierung gehen. Menschen beispielsweise mit psychischen Erkrankungen sind in ihrer Subjekthaftigkeit nicht anders zu sehen als andere Patienten. Sie gehören zur Gruppe jener Erkrankten, bei denen gesellschaftliche Vorurteile besonders rasch ausgelöst werden. Aber sie haben auch bevorzugte Chancen, bei der Entwicklung ihrer Selbstheilungskräfte ein differenziertes Bewusstsein über ihre Erkrankung und deren Verschränktheit mit den sozialen Umfeldbedingungen zu erwerben.

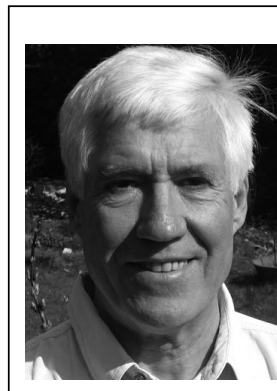

Hansgeorg Ließem ist freier Sozialplaner mit Projekterfahrungen in der Sozialpsychiatrie seit 1978. Er war Berater und Projektentwickler für mehrere Vorhaben der Integrierten Psychiatrischen Versorgung in Deutschland und Leiter von Arbeitskreisen zur Ausarbeitung von psychiatrischen

Projekten der Integrierten Versorgung in mehreren Bundesländern in Zusammenarbeit mit dem Verein für Soziotherapie e. $V$. E-Mail liessem@t-online.de

Wenn ich mich im Weiteren mit der Frage beschäftige, wie sich Projekte der Integrierten Versorgung in der Psychiatrie aus Patientensicht bewerten lassen, dann gehe ich grundsätzlich davon aus, dass der psychisch Erkrankte in jeder Phase seiner Erkrankung fähig ist, Entscheidungen zu treffen und damit seine Subjekt-Rolle wahrzunehmen. Zweifellos gibt es zahlreiche Möglichkeiten, diese Entscheidungsfähigkeit zumindest zeitweise einzuschränken: durch Zwangsmaßnahmen, durch Medikamente, durch sozialen Druck. Doch die immer noch verbreitete Grundauffassung, eine schwere psychische Erkrankung sei grundsätzlich mit dem Verlust von subjekthafter Entscheidungskompetenz verbunden, wurde durch die vom Bundesministerium für Gesundheit geförderten beiden Forschungsprojekte in Freiburg und München widerlegt. Sie zeigen, dass auch schwer depressiv und hochpsychotisch Erkrankte in der Lage sind, Entscheidungen über die weitere Behandlung zu treffen, wenn man ihnen entsprechende Alternativen vorstellt. (3) Es macht also Sinn, bei der Entwicklung einer neuen Arbeitsstruktur in der Psychiatrie nach den Kriterien zu fragen, nach denen Patienten aus ihrer Interessenlage heraus die Entwicklung beurteilen könnten.

Welche Fragestellungen müssen die Projekte der Integrierten Versorgung in der Psychiatrie erwarten, von deren Berücksichtigung Patienten ihre Zufriedenheit abhängig machen?

\section{Vorrang ambulanter Behandlung}

Mit der Aufnahme eines Patienten in das Behandlungssystem einer Integrierten Versorgung übernimmt der Patient die Verpflichtung, seine weitere Behandlung von den 
Leistungserbringern in Anspruch zu nehmen, die sich zu dieser Versorgungskette verbunden haben. Der Patient geht daher eine Bindung ein zu einem Zeitpunkt, an dem er die Leistungsfähigkeit der beteiligten Leistungserbringer noch nicht einschätzen kann. Um dennoch eine qualifizierte Entscheidung zu treffen, benötigt er vorausgehende Informationen, die ihm zumindest seine Frage beantworten, welches Grundkonzept von Behandlung und Begleitung von den Beteiligten verfolgt wird.

Aus Patientensicht geht es dabei vorrangig um die Frage, ob die Integrierte Versorgung einen ambulanten Schwerpunkt besitzt oder eine stationäre Versorgung im Fokus hat. Wird das integrierte Angebot von einem Krankenhaus aus organisiert, bei dem ambulante Behandlungsinstanzen als »komplementär« angesehen werden, so muss der Patient davon ausgehen, dass intensive Behandlungsphasen vorrangig stationär durchgeführt werden. Ein erheblicher Teil der Patientenschaft will aber Krankenhausaufenthalte vermeiden, weil sie mit erheblichen Nachteilen für die soziale und berufliche Einbindung verbunden sind.

\section{Was will die Anamnese leisten?}

Jede medizinische Behandlung beginnt typischerweise mit der Anamnese. Die Arztfrage »Wo tut's denn weh?« versucht die Symptomatik zu lokalisieren, kann aber aus Patientensicht nur der Beginn einer intensiven Bemühung sein, die Entstehungsgeschichte der Symptomatik aus der individuellen seelisch-körperlichen Verfassung und im Kontext mit dem sozialen und räumlichen Umfeld zu erarbeiten. »Warum geht's mir so schlecht?«, ist eine Frage, die nur in einem komplizierten Gedanken- und Gesprächsprozess aufgearbeitet werden kann. Das setzt auf beiden Seiten Vertrauen voraus und Beziehungs- und damit Behandlungskontinuität.

Für die Integrierte Versorgung stellt sich daher die Frage, welchen Stellenwert und welche Ausgestaltung die Anamnese erfährt. Ist sicher gestellt, dass die therapeutischen Behandlungsmaßnahmen immer in einen Zusammenhang mit der Anamnese gestellt werden, werden sie gegenüber dem Patienten offen gelegt und mit ihm abgestimmt? Stehen überhaupt therapeutische Grundfragen am Anfang der integrierten Behandlung?

Nicht im Sinne der Patienten wäre eine Anamnese-Praxis, bei der lediglich die bisherige Medikation abgefragt würde, um darauf eigene Pharmaverordnungen aufzubauen. Es kann nicht nur um die Verträglichkeit bestimmter Medikamente gehen und die subjektive Einstellung gegenüber deren Nebenwirkungen. Wenn sich Patienten dagegen verwahren, dass Gespräche durch die Verordnung von Psychopharmaka ersetzt werden, dann hat dies wenig mit fehlender »Compliance« (4) zu tun.

\section{Einbeziehung des Patienten in den Behandlungsablauf}

Viele Patienten wünschen sich eine aktive Einbeziehung in den Behandlungsablauf. Diese Einbeziehung setzt zwingend voraus, dass die beteiligten Fachleute die Notwendigkeit zur Ausarbeitung von Behandlungsalternativen anerkennen. In diesem Zusammenhang gilt es, die professionelle Angewohnheit zu überwinden, die eigene Unsicherheit über den richtigen nächsten Schritt als Schwäche und mangelnde Professionalität anzusehen. Wer als Arzt, Therapeut oder Sozialarbeiter nicht gänzlich in professioneller Routine erstarrt ist, der erlebt immer wieder eigene Entscheidungsnot. Aus Patientensicht bietet diese Entscheidungsnot, wenn sie dazu führt, die denkbaren Alternativen zu benennen, die konkrete Chance, die eigenen Gedanken und Gefühle in die Suche nach dem besten nächsten Schritt mit einzubringen.

\section{Alternativen zur pharmakologischen Therapie}

Die Patientenmeinung zur pharmakologischen Therapie ist sehr unterschiedlich. Sie reicht von entschiedener Ablehnung über differenzierte Zustimmung bis zur erzwungenen Hinnahme. Kaum ein Patient findet ein medizinisches Angebot ausreichend, wenn es nur aus der Verordnung von Medikamenten besteht. Tritt ein Projekt der Integrierten Versorgung mit dem Anspruch auf, eine interessante Variante zur herkömmlichen Psychiatrie anzubieten, wird sie mit Alternativen zur pharmakologischen Therapie aufwarten müssen. Die Patientenbedenken richten sich dabei nicht nur auf die unerwünschten Nebenwirkungen der Medikamente, sondern auch gegen die durch Medikamente hervorgerufenen Einschränkungen in der freien Willensbildung und damit der Fähigkeit, sich an der Therapie aktiv zu beteiligen.

\section{Soziotherapie und ambulante Pflege}

Bei Menschen mit einer psychischen Erkrankung kann nicht grundsätzlich unterstellt werden, dass sie eine Behandlung anstreben, die in jeden privaten Lebensbereich eingreift. Dies gilt auch dann, wenn wichtige Lebensfelder von und durch die Erkrankung negativ tangiert werden. Die Behandlung muss aber grundsätzlich in der Lage

\section{Anmerkungen}

(1) Siehe hierzu: Hansgeorg Ließem (Hg.): Integrierte Versorgung in der Gemeindepsychiatrie, Bonn 2007 (zu beziehen über den Dachverband Gemeindepsychiatrie).

(2) Der Autor gibt im Nachfolgenden Gesprächserfahrungen wieder, die er in den letzten Jahren mit vielen betroffenen Menschen machen konnte. Außerdem fällt es nicht allzu schwer, sich selbst in die Situation eines Psychiatrie-Patienten zu versetzen. So weit entfernt liegen weder psychische Krisen noch die Abhängigkeit von medizinisch begründeten Entscheidungen.

(3) Nähere Informationen zum BMG-Förderschwerpunkt »Patient als Partner im medizinischen Entscheidungsprozess « und zu den Projekten in Freiburg und München im Internet: http://www.patient-als-partner.de.

(4) Ein häufig benutzter englischer Begriff, den man auch mit »Willfährigkeit« übersetzen könnte. Aus Patientensicht sollte er durch den Begriff »Zusammenarbeit « ersetzt werden, deren Qualität immer von mindestens zwei Partnern abhängt. 
sein, in das gesamte Lebensumfeld des Patienten hineinwirken zu können. Die Ausdehnung des Wirkungskreises darf nur mit dem Patienten gemeinsam geschehen, dessen Selbstständigkeit und Handlungskompetenz es zu steigern gilt. Die gegebenenfalls notwendige Unterstützung bedingt einmal eine koordinierende Unterstützung (Soziotherapie) sowie zum andern eine stabilisierende Alltagsbegleitung (ambulante häusliche Pflege).

Aus Patientensicht sind daher Soziotherapie und Ambulante Psychiatrische Pflege unabdingbare Kernelemente jeder integrierten psychiatrischen Versorgung. Für beide Aufgabenbereiche sind ausreichende Zeitkontingente bereitzustellen sowohl hinsichtlich der Dauer des Einzeleinsatzes wie auch in Bezug auf die Langfristigkeit der Verordnung.

\section{Krisenmanagement}

Patienten wünschen sich bei Integrierter Versorgung, dass sie gerade bei auftretenden Krisen ihre ambulante Tauglichkeit erweist. Krisensicher ist eine Versorgung, wenn der Bezugstherapeut für »seine« Patienten per Handy immer erreichbar ist. Wenn zur Krisenbewältigung rasche Maßnahmen gehören, sollten diese vordringlich eine ambulante Form besitzen und nicht die Krankenhauseinweisung als Standardlösung.

Jede Integrierte Versorgung sollte daher in ihrer Selbstdarstellung Aussagen zum Notfallmanagement treffen, die dem Patienten vor seiner Aufnahme in das Versorgungssystem ausgehändigt werden. Er muss die Chance haben, sich zu den vorgestellten Möglichkeiten und Maßnahmekriterien ein eigenes Bild zu machen. Es bietet sich an, gerade für den Umgang in extremen Krisensituationen eine Vereinbarung mit dem Patienten zu schließen, die den Rahmen festlegt, in dem im potenziellen Falle die notwendigen Entscheidungen getroffen werden können.

\section{Niederschwelliges Beschwerde-Management}

Es erfordert einiges Selbstbewusstsein, um sich im Gesundheitswesen über vermeintliches oder tatsächliches Fehlverhalten der beteiligten Profis zu beschweren. Die Furcht ist gar nicht so abwegig, dass sich durch eine Beschwerde das Gesprächsklima mit den beteiligten Fachkräften verschlechtert. Besonders in der Psychiatrie wird die Patientenbeschwerde gerne in die Schublade mangelnder Krankheitseinsicht abgelegt und damit dem Patienten negativ angelastet.

In einer auf Qualitätssteigerung ausgerichteten Integrierten Versorgung ist Kritik von Patienten ein wichtiger Baustein zur Verbesserung des Angebotes und der fachlichen Arbeitsweise. Die Frage kann also nicht sein, ob die Integrierte Versorgung Kritik zulässt, sondern sie muss lauten: Was tut sie, damit sich auch die besonders Ängstlichen trauen, ihre Kritik vorzutragen? Vieles wird sich positiv gestalten lassen im Zuge einer wirklich vertrauensvollen Zusammenarbeit zwischen den Patienten und ihren Bezugstherapeuten. Doch Vertrauen ersetzt nicht immer fehlenden Mut gegenüber Personen, von denen man sich letztlich abhängig fühlt.

\section{Was ist eigentlich Integrierte Versorgung?}

Der Begriff bezeichnet auf der politischen und betrieblichen Ebene (die Schaffung von) Versorgungsnetzwerke(n), in denen an einem Ort oder in einer Region die Leistungserbringer der stationären, ambulanten und rehabilitativen Versorgung im Gesundheits- und Sozialwesen in geregelter Weise ergebnisorientiert zusammenarbeiten. Auf der Mikroebene des Einzelfalls richtet sich Integrierte Versorgung auf die Einbeziehung einzelner humandienstlicher Leistungen in das Ganze eines personenbezogenen Versorgungszusammenhangs.

Die Integration der Leistungserbringung erfolgt organisatorisch durch den Aufbau geeigneter Strukturen, zum Beispiel in Verbundsystemen der Jugendhilfe, die in einem Sozialraum etabliert werden, oder über Servicestellen im System der Rehabilitation behinderter Menschen, und sie kommt fachlich durch interdisziplinäre Kooperation zustande. Hauptsächlich wird von Integrierter Versorgung im Gesundheitswesen gesprochen. Medizinische Behandlung soll möglichst bruchlos und in Überwin- dung von Sektorgrenzen und Kommunikationsbarrieren erfolgen. Zur Überwindung der vorhandenen Trennungen und zur Lösung von Schnittstellenproblemen in Behandlungs- und Versorgungsabläufen ist ein Schnittstellenmanagement bzw. ein Überleitungsmanagement erforderlich. Eine Integrierte Versorgung wird in der Reform des Gesundheitswesens und in verschiedenen Bereichen der Erbringung von Sozialleistungen angestrebt. Integrierte Versorgung erfordert eine sektor- und fachübergreifende Zusammenarbeit von Akteuren. Für das medizinische Versorgungssystem stellen die im GKV-Modernisierungsgesetz (GMG) 2004 neu gefassten §§ 140 a-d SGB V die leistungsrechtliche und vertragsrechtliche Grundlage für eine Integrierte Versorgung dar. Der Ausgestaltung von Integrationsverträgen ist viel Spielraum gelassen. Die gesetzliche Krankenkasse kann »Verträge über eine verschiedene Leistungssektoren übergreifende Versorgung der Versicherten oder eine interdisziplinär-fachübergreifende Versorgung « abschließen (§ 140 a SGB V). Einer Krankenkasse bietet sich die Möglichkeit, außerhalb der für die Regelversorgung geltenden Kollektivverträge mit den Kassenärztlichen Vereinigungen direkt Einzelverträge mit Leistungserbringern über integrierte Versorgungsformen abzuschließen.

(...)

Analog sieht das SGB IX zur Rehabilitation eine Integrierte Versorgung vor. Sie bedeutet eine Abstimmung aller für eine Person benötigten Dienste aufeinander und die strukturelle Bahnung dieser Integration in der Leistungserbringung. In den Verträgen zur Integrierten Versorgung müssen sich »die Vertragspartner der Krankenkassen zu einer qualitätsgesicherten, wirksamen, ausreichenden, zweckmäßigen und wirtschaftlichen Versorgung der Versicherten verpflichten « (§ 140 b Abs. 3 SGB V).

Ein Krankenhaus expandiert bei Ausgestaltung Iintegrierter Versorgung in das ambulante Leistungsangebot. Es kann auf der Grundlage eines Integrationsvertrages gemäß § 140 b SGB V mit niedergelassenen Ärzten und anderen Gesundheitsdiensten kooperieren und zum Systemmanager der ganzen lokalen Gesundheitsversorgung werden.

(...)

Eine weitere Form der Integrierten Versorgung stellt das Medizinische Versorgungszentrum (gem. § 95 SGB V) dar, das fachübergreifend mit Vertragsärzten oder angestellten Ärzten und mit komplementären Diensten eine lokale ambulante Versorgung übernimmt. Das Behandlungsangebot muss mindestens zwei medizinische Fachrichtungen umfassen.

(...)

Auf der politisch-ökonomischen Makroebene geht es bei der Integrierten Versorgung um die Steuerung der Leistungserbringung sektorübergreifend in korporativer Koordination. Dabei wird über die Bereiche der Sozial-, Gesundheits-, Bildungs- und Wirtschaftspolitik hinweg nach Verbundlösungen gesucht, mit denen eine ökonomisch vertretbare und gegenüber segregierten Strukturen bessere Versorgung der Bevölkerung erreicht wird.

Wolf Rainer Wendt

Quelle: Bernd Maelicke (Hg.): Lexikon der Sozialwirtschaft. Nomos Verlagsgesellschaft, Baden-Baden 2007. 1.128 Seiten. 98,- Euro. ISBN 978-3-8329-2511-6. Seite $507 \mathrm{f}$. 